\title{
Towards Automatic Generation of Conceptual Interpretation of Clustering ${ }^{\star}$
}

\author{
Alejandra Pérez-Bonilla ${ }^{1,2}$ and Karina Gibert ${ }^{1}$ \\ ${ }^{1}$ Dep. of Statistics and Operations Research, Technical University of Catalonia. \\ Campus Nord; Edif. C5. C/ Jordi Girona 1-3; 08034 Barcelona, Spain \\ ${ }^{2}$ Department of Industrial Engineering, University of Santiago of Chile \\ Avda. Ecuador 3769, Santiago, Chile. \\ \{alejandra.perez, karina.gibert\}@upc.edu
}

\begin{abstract}
In this paper the Methodology of conceptual characterization by embedded conditioning $C C E C$, oriented to the automatic generation of conceptual descriptions of classifications that can support later decisionmaking is presented, as well as its application to the interpretation of previously identified classes characterizing the different situations on a WasteWater Treatment Plant (WWTP). The particularity of the method is that it provides an interpretation of a partition previously obtained on an ill-structured domain, starting from a hierarchical clustering. The methodology uses some statistical tools (as the boxplot multiple, introduced by Tukey, which in our context behave as a powerful tool for numeric variables) together with some machine learning methods, to learn the structure of the data; this allows extracting useful information (using the concept of characterizing variable) for the automatic generation of a set of useful rules for later identification of classes. In this paper the usefulness of $C C E C$ for building domain theories as models supporting later decision-making is addressed.
\end{abstract}

Keywords: Hierarchical clustering, class interpretation, Knowledge Discovery and Data Mining.

\section{Introduction}

In automatic classification where the classes composing a certain domain are to be discovered, one of the most important required processes and one of the less standardized one, is the interpretation of the classes, closely related with validation 1 and critical in the later usefulness of the discovered knowledge. The interpretation of the classes, so important to understand the meaning of the obtained classification as well as the structure of the domain, use to be done in an artistic-like way. But this process becomes more and more complicate as the number of classes grows. This work tries to face the problem of the automatic generation of useful interpretations of classes in such a way that decisions about the treatment of a new object can be modelled, to develop, in the long term, a software tool which supports decision-making.

\footnotetext{
^ This research has been partially financed by the project TIN 2004-01368.
} 
Conceptual characterization by embedded conditioning $(C C E C)$ [2] is the proposal presented in this paper to cover these goals. Given a clustering, $C C E C$ provides an automatic interpretation for it that supports the later construction of intelligent decision support systems for assisting decision-making. A particular application to WWTP is addressed. The presented proposal integrates different findings from a series of previous works [3] 4] in a single methodological tool which takes advantage of the hierarchical structure of the clustering to overcome some of the limitations observed in [3], [5]. The application is presented in next section in order to be used for illustration in 93

This paper is organized as follows: After the introduction, WWTP are presented in $₫ 2$ as well as, the description of the specific data base that has been analyzed, the basic concepts are in $\$ 3$. The $\$ 4$ presents the proposed methodology $C C E C$ and the results of applying $C C E C$ to the data described in $\$ 5$. Finally in 86 the conclusions and the future work are addressed.

\section{Application}

In this paper a real WWTP is used to validate the proposed methodology 4 . First, a very brief description of the general WWTP process is briefly introduced the water flows sequentially through three or four processes (see 6 for a detailed description of the process): ( $i$ ): In the pretreatment, an initial separation of solids from wastewater is performed. (ii) Primary treatment consists of leaving the wastewater in a settler for some hours. Solids will deposit down the settler and could be sent out. (iii) Secondary treatment occurs inside a biological reactor; a population of microorganisms (biomass) degrades the organic matter solved in the wastewater. (iv) In the advanced treatment another settler is used to separate the water from the biomass. The settler output (solids or biomass) produces a kind of mud which is the input of another process called sludge line. Next the plant used in this research described is a WWTP is located in Catalunya (Spain). Data analyzed in this work is a sample of 396 observations taken from September the first of 1995 to September the 30th of 1996. Each observation refers to a daily mean, and it is identified by the date itself.

The state of the Plant is described through a set of 25 variables, considered the more relevant upon expert's opinions. They can be grouped as: (i) Input (measures taken at the entrance of the plant): Q-E: Inflow wastewater (daily $m^{3}$ of water);FE-E Iron pre-treatment (g/l); pH-E; SS-E: Suspended Solids (mg/l); SSV-E: Volatile suspended solids (mg/l); COD-E: Chemical organic matter (mg/l); BOD-E: Biodegradable organic matter (m/l). (ii) After Settler (measures taken when the wastewater comes out of the first settler): PH-D: pH; SS-D: Suspended Solids (mg/l); SSV-D: Volatile suspended solids (mg/l); COD-D: Chemical organic matter (mg/l); BOD-D: Biodegradable organic matter $(\mathrm{m} / \mathrm{l})$. (iii) Biological treatment (measures taken in the biological reactor): Q-B: Biological reactor-flow; V30: Index at the biological reactor (measures the sedimentation quality of the mixed liquor, in ml/l); MLSS-B: Mixed liquor suspended solids at the biological reactor; MLVSS-B: Mixed liquor volatile 
suspended solids: MCRT-B: Mean cell residence time at the biological reactor. (iv) Output (when the water is meeting the river): PH-S: pH ; SS-S: Suspended Solids (mg/l); SSV-S: Volatile suspended solids (mg/l); COD-S: Chemical organic matter $(\mathrm{mg} / \mathrm{l})$; BOD-S: Biodegradable organic matter $(\mathrm{m} / \mathrm{l})$. (v) Other variables: QR-G: Recirculated Flow ; QP-G: Purged flow; QA-G: Air inflow.

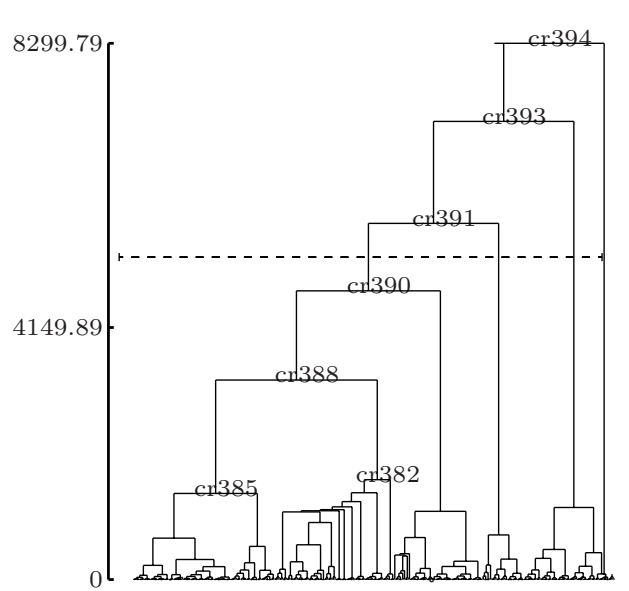

The standard input of a clustering algorithm use to be a data matrix with the values of $K$ variables $X_{1} \ldots X_{K}$ (numerical or not) observed over a set $\mathcal{I}=\{1, \ldots n\}$ of individuals. Variables are in columns, while individuals in rows. Cells contain the value $\left(x_{i k}\right)$, taken by individual $i \in \mathcal{I}$ for variable $X_{k},(k=$ $1: K)$. The set of values of $X_{k}$ is named $\mathcal{D}^{k}=\left\{c_{1}^{k}, c_{2}^{k}, \ldots, c_{s}^{k}\right\}$ for categorical variables and $D^{k}=r_{k}$ for numerical ones, being $r_{k}$ $=\left[\min X_{k}, \max X_{k}\right]$ the range of $X_{K}$. A partition in $\xi$ classes of $\mathcal{I}$ is denoted by $\mathcal{P}_{\xi}=$ $\left\{C_{1}, \ldots, C_{\xi}\right\}$, and $\tau=\left\{\mathcal{P}_{1}, \mathcal{P}_{2}, \mathcal{P}_{3}, \mathcal{P}_{4}, \ldots, \mathcal{P}_{n}\right\}$ is an indexed hierarchy of $\mathcal{I}$. Finally, $\mathcal{P}_{2}=$ $\left\{C_{1}, C_{2}\right\}$ is a binary partition of $\mathcal{I}$. Usually, $\tau$ is the result of a hierarchical clustering over $\mathcal{I}$, and it can be represented in a graphical way in what is known as a dendrogram (or hierarchical tree, see figure 1, [4]).

Fig. 1. Left: Hierarchical tree $\left[\tau_{G i 2, R 1}^{E n, G}\right][2]$; Right: Notation

The data base previously presented, was classified in a previous work [4 by using clustering based on rules [3, producing the hierarchical tree of Figure 11(left).

The Figure 2 contains the class panel graph [7] of the 25 variables regarding the top partition $\mathcal{P}_{2}=\left\{C_{393}, C_{392}\right\}$ where the multiple boxplot 8 ] of variables for each class are displayed. As usual in hierarchical clustering, the final partition is the horizontal cut of the tree that maximizes the ratio between heterogeneity between classes with respect to homogeneity within classes, what guaranties the distinguishability of classes. The result is a 4-classes partition $\mathcal{P}_{4}=\left\{C_{392}, C_{389}, C_{390}, C_{383}\right\}$.

\section{Basic Concepts}

Four main concepts are in the kernel of this work (basic notation is introduced in Figure 1(right)):

- Variable $X_{k}$ is Totally characterizing of class $C \in \mathcal{P}$, if all the values taken by $X_{k}$ in class $C$ are characteristic of $C$ (objects of other classes take other values). As an example, see variables Q-E or QR-G in Figure 2.

- Variable $X_{k}$ is Partially characterizing of class $C \in \mathcal{P}$ if there is at least one characteristic value of $C$, although the class can share some other values 


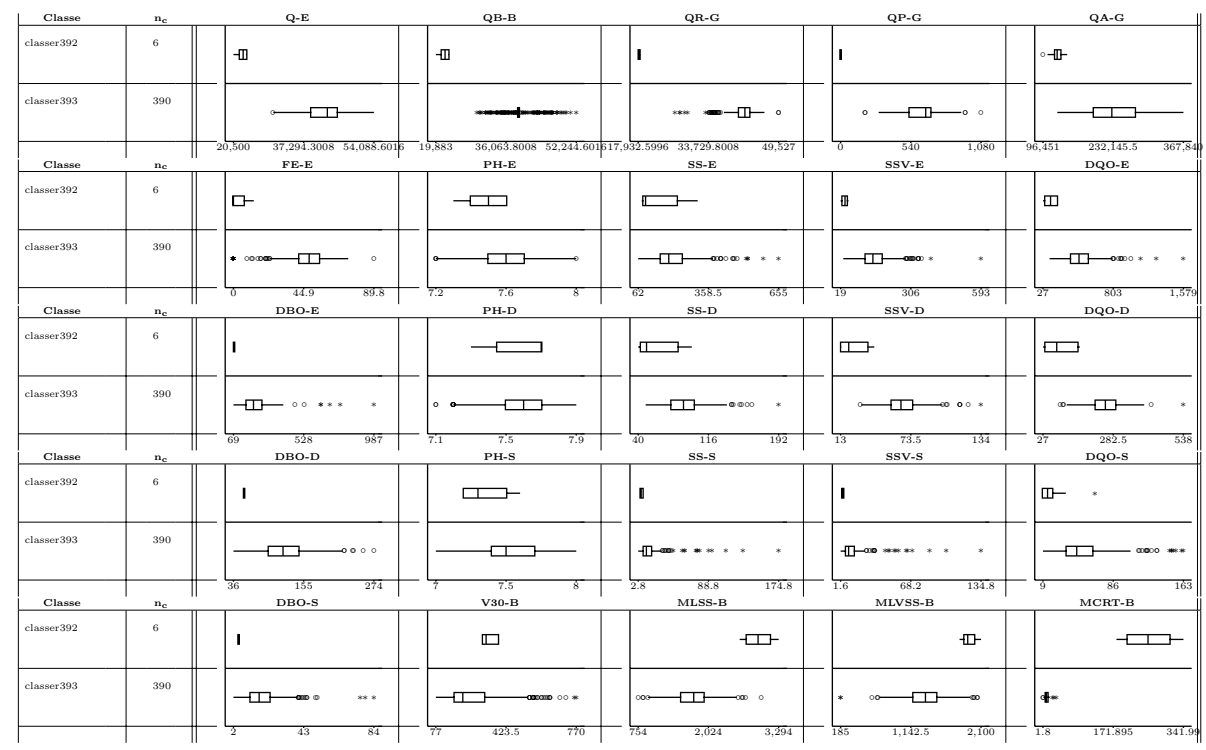

Fig. 2. class panel graph of $\mathcal{P}_{2}$

with other classes. See PH-E in Figure 2 which is partially characterizing the class $C_{392}$ if it takes very low or high values.

- Covering (Cov): Given a rule $r: x_{i k} \in I_{s}^{k} \longrightarrow C$ the covering of $r$ is the number of objects in $\mathcal{I}$ that satisfy the rule. $\operatorname{Cov}(r)=\operatorname{card}\left\{i \in C \operatorname{tq} \quad x_{i k} \in I_{s}^{k}\right\}$

- Relative covering $(\operatorname{Cov} R)$ : Given a rule, the relative covering is the proportion of of class $\mathrm{C}$ that satisfy the rule. $\operatorname{Cov} R(r)=\frac{\operatorname{card}\left\{i \in C \quad t q \quad x_{i k} \in I_{s}^{k}\right\}}{\operatorname{card}\{C\}} * 100$

Boxplot based discretization (BbD) is presented in [2, 9] and revised in [10, as an efficient way of transforming a numerical variable into a qualitative one in such a way that the cut points for discretizing identify where the subsets of intersecting classes between classes change and, given $X_{k}, \mathcal{P}$, it consists of the following steps:

1. Calculate the minimum $\left(m_{C}^{k}\right)$ and maximum $\left(M_{C}^{k}\right)$ of $X_{k}$ inside any class. Built $\mathcal{M}^{k}=\left\{m_{C_{1}}^{k}, \ldots, m_{C_{\xi}}^{k}, M_{C_{1}}^{k}, \ldots, M_{C_{\xi}}^{k}\right\}$, where $\operatorname{card}\left(\mathcal{M}^{k}\right)=2 \xi$

2. Built the set of cutpoints $\mathcal{Z}^{k}$ by sorting $\mathcal{M}^{k}$ in increasing way into $\mathcal{Z}^{k}=$ $\left\{z_{i}^{k} ; i=1: 2 \xi\right\}$. At every $z_{i}^{k}$ the intersection between classes changes arity.

3. Built the set of intervals $I^{k}$ induced by $\mathcal{P}$ on $X_{k}$ by defining an interval $I_{s}^{k}$ between every pair of consecutive values of $\mathcal{Z}^{k} . \mathcal{I}^{k}=\left\{I_{1}^{k}, \ldots, I_{2 \xi-1}^{k}\right\}$ is the boxplot based discretization of $X_{k}$. The $I_{s}^{k}, s \in\{1,2 \xi-1\}$, intervals are variable length and the intersection among classes changes from one to another.

As an example, Table1(left) contains descriptive statistics of Q-E versus $\mathcal{P}_{2}$. In the right hand side of Table 1 the set of minimums and maximums of every class 
Table 1. (left): Summary statistics for Q-E vs $\mathcal{P}_{2} ;$ (right): $\mathcal{M}^{Q-E}$, Set of extreme values of $\mathrm{Q}-\mathrm{E} \mid \mathcal{C} \in \mathcal{P}_{2}$ and $\mathcal{Z}^{Q-E}$, corresponding ascendant sorting

\begin{tabular}{|c|c||c|c|}
\hline & Class & C393 & C392 \\
\hline Var. & $\mathrm{N}=396$ & $n_{c}=390$ & $n_{c}=6$ \\
\hline \hline \multirow{3}{*}{ Q-E } & $\overline{\mathbf{X}}$ & $42,112.9453$ & $22,563.7988$ \\
\cline { 2 - 4 } & $\mathbf{S}$ & $4,559.2437$ & $1,168.8481$ \\
\cline { 2 - 4 } & $\mathbf{m i n}$ & 29,920 & 20,500 \\
\cline { 2 - 4 } & $\mathbf{m a x}$ & $54,088.6016$ & $23,662.9004$ \\
\cline { 2 - 4 } & $\mathbf{N}^{*}$ & 1 & 0 \\
\hline
\end{tabular}

\begin{tabular}{|c|c|}
\hline $\mathcal{M}^{Q-E}$ & $\mathcal{Z}^{Q-E}$ \\
\hline \hline 29,920 & 20,500 \\
\hline 20,500 & $23,662.9004$ \\
\hline $54,088.6016$ & 29,920 \\
\hline $23,662.9004$ & $54,088.6016$ \\
\hline
\end{tabular}

$\mathcal{M}^{Q-E}$ is sorted into $Z^{Q-E}$ and its values can be considered as the cut points of a discretization of Q-E in 3 intervals: $I^{Q-E}=\left\{I_{1}^{Q-E}, I_{2}^{Q-E}, I_{3}^{Q-E}\right\}$, with $I_{1}^{Q-E}=$ $[20500.0,23662.9], I_{2}^{Q-E}=(23662.9,29920.0), I_{3}^{Q-E}=[29920.0,54088.6]$. Thus, $I^{Q-E}$ is a discretization of $X_{k}$ which takes 3 values. Only elements of $C_{392}$ can take value $I_{1}^{Q-E}$. Only elements of $C_{393}$ take value $I_{3}^{Q-E}$ and $I_{2}^{Q-E}$ is empty. For variable $I^{S S-S}=\left\{I_{1}^{S S-S}, I_{2}^{S S-S}, I_{3}^{S S-S}\right\}$, with $I_{1}^{S S-S}=[2.80,3.200), I_{2}^{S S-S}=$ $[3.20,20.00], I_{3}^{S S-S}=(20.0,174.8]$. Only elements of $C_{391}$ can take value $I_{1}^{S S-S}$; along $I_{2}^{S S-S}, C_{391}$ is intersecting with $C_{389}$ and along $I_{3}^{S S-S}$ does not intersect. The values of $\mathcal{M}^{k}$ are identifying the points where intersections among classes change.

The methodology boxplot based induction rules (BbIR) is presented in [9]. It is a method for generating probabilistic concepts with a minimum number of attributes in the antecedent, using of the boxplot based discretization of $X_{k}$.

1. Use the boxplot based discretization to build $r_{s}: \mathcal{I}^{k}=\left\{I_{1}^{k}, I_{2}^{k}, I_{3}^{k}, \ldots, I_{2 \xi-1}^{k}\right\}$.

2. Build table $I^{k} \times P$ where rows are indexed by $s \in\{1,2 \xi-1\}$, and columns by $C \in\{1, \xi\}$.

3. Built the table $I^{k} \mid \mathcal{P}$ by dividing the cells of $I^{k} \times \mathcal{P}$ by the row totals. Cells, $p_{s c}=P\left(C \mid I^{k}=I_{s}^{k}\right)=P\left(i \in C \mid x_{i k} \in I_{s}^{k}\right)=\frac{\operatorname{card}\left\{i: x_{i k} \in I_{s}^{k} \wedge i \in C\right\}}{\operatorname{card}\left\{i \in \mathcal{I}: x_{i k} \in I_{s}^{k}\right\}}=\frac{n_{s c}}{\sum_{\forall s} n_{s c}}$ If $\exists C: p_{s c}=1 \& \operatorname{Cov}\left(r_{s}\right)=100 \%$, then $X_{k}$ is a totally characterizing variable of $C$.

4. For every cell in table $I^{k} \mid \mathcal{P}$ produce the rule: If $x_{i k} \in I_{s}^{k} \stackrel{p_{s c}}{\longrightarrow} i \in C$ For example, see Table 2(left) $I^{Q-E} \times P_{2}$, since $\operatorname{card}\left\{i \in \mathcal{I}: x_{i k} \in I_{1}^{Q-E}\right\}=$ $\operatorname{card}\left\{i \in \mathcal{I}: i \in C_{392}\right\}=\operatorname{card}\left\{i \in \mathcal{I}: x_{i k} \in I_{1}^{Q-E} \wedge i \in C_{392}\right\}=6$. Then $p_{1 C_{392}}=1$ and the rule: $x_{Q-E, i} \in(23662.9,29920.0] \stackrel{1.0}{\longrightarrow} C_{392}$ is generated.

From $I^{Q-E} \mid \mathcal{P}_{2}$ (see Table 2 (center)) the following system of probabilistic rules, can be induced for the variable $\mathrm{Q}-\mathrm{E}$ :

$$
\begin{aligned}
& r_{1, \text { classer } 393}^{Q-E}: x_{Q-E, i} \in[20500.0,23662.9] \stackrel{0.0}{\longrightarrow} \text { classer } 393 \\
& r_{1, \text { classer } 392}^{Q-E}: x_{Q-E, i} \in[20500.0,23662.9] \stackrel{1.0}{\longrightarrow} \text { classer } 392 \\
& r_{3, \text { classer } 393}^{Q-E}: x_{Q-E, i} \in[29920.0,54088.6] \stackrel{1.0}{\longrightarrow} \text { classer } 393 \\
& r_{3, \text { classer } 392}^{Q-E}: x_{Q-E, i} \in[29920.0,54088.6] \stackrel{0.0}{\longrightarrow} \text { classer } 392
\end{aligned}
$$

Finally too more concepts are used in this work. 
Table 2. (left): $I^{Q-E} \times P_{2} ;($ center $): I^{Q-E} \mid \mathcal{P}_{2}$ table; (right): $\mathcal{P}_{2} \times \mathcal{P}_{3}$

\begin{tabular}{|c|c|c|}
\hline & $C_{392}^{2}$ & $C_{393}^{2}$ \\
\hline$I_{1}^{Q-E}$ & 6 & 0 \\
\hline$I_{2}^{Q-E}$ & 0 & 0 \\
\hline$I_{3}^{Q-E}$ & 0 & 389 \\
\hline
\end{tabular}

\begin{tabular}{|l|c|c|}
\hline & $C_{392}^{2}$ & $C_{393}^{2}$ \\
\hline$I_{1}^{Q-E}$ & 1 & 0 \\
\hline$I_{2}^{Q-E}$ & 0 & 0 \\
\hline$I_{3}^{Q-E}$ & 0 & 1 \\
\hline
\end{tabular}

\begin{tabular}{|l|c|c|}
\hline$P_{3}$ vs $P_{2}$ & $C_{393}^{2}$ & $C_{392}^{2}$ \\
\hline$C_{392}^{3}$ & 0 & 6 \\
\hline$C_{391}^{3}$ & 320 & 0 \\
\hline$C_{389}^{3}$ & 70 & 0 \\
\hline
\end{tabular}

\section{The Metodology}

$C C E C$ takes advantage of the existence of $\tau$, and uses the property of any binary hierarchical structure that $\mathcal{P}_{\xi+1}$ has the same classes of $\mathcal{P}_{\xi}$ except one, which splits in two subclasses in $\mathcal{P}_{\xi+1}$. Binary hierarchical structure will be used by $C C E C$ to discover particularities of the final classes step by step also in hierarchical way. The $C C E C[2]$ allows generation of automátic conceptual interpretations of a given partition $\mathcal{P} \in \tau$. The steps to be followed are described bellow. The application to the WWTP is illustrated in section $\$ 5$

1. Cut the tree at highest level (make $\xi=2$ and consider $\mathcal{P}_{2}=\left\{C_{1}, C_{2}\right\}$ ).

2. Use the boxplot based discretization presented in [9] and revised in [10], to find (total or partial) characteristic values for numerical variables 5 .

3. Use boxplot based induction rules (BbIR), to induce a the knowledge Base describing both classes.

4. For classes in $\mathcal{P}_{2}$, determine concepts $A_{1}^{\xi, X_{k}}:$ " $\left[X_{k} \in I_{s}^{k}\right]$ ", $A_{2}^{\xi, X_{k}}: \neg A_{1}^{\xi, X_{k}}$ associated to $C_{1}, C_{2}$, by taking the intervals provided by a totally characteristic variable or the partial one with greater relative covering and $p_{s c}=1$.

5. Go down one level in the tree, by making $\xi=\xi+1$ and so considering $\mathcal{P}^{\xi+1}$. As said before $\mathcal{P}^{\xi+1}$ is embedded in $\mathcal{P}^{\xi}$ in such a way that there is a class of $\mathcal{P}^{\xi}$ split in two new classes of $\mathcal{P}^{\xi+1}$, namely $C_{i}^{\xi+1}$ and $C_{j}^{\xi+1}$ and all other classes are common to both partitions.

Since in the previous step $C_{i}^{\xi+1} \cup C_{j}^{\xi+1}$ were conceptually separated from the rest, at this point it is only required to find the variables which separate (or distinguishes) $C_{i}^{\xi+1, X_{k}}$ from $C_{j}^{\xi+1, X_{k}}$, by repeating steps 2-4. Suppose $B_{i}^{\xi+1, X_{k}}$ and $B_{j}^{\xi+1, X_{k}}$ the concepts induced from $C_{i}^{\xi+1, X_{k}}$ and $C_{j}^{\xi+1, X_{k}}$, in the step $\xi+1$.

6. Integrate the extracted knowledge of the iteration $\xi+1$ with that of the iteration $\xi$, by determining the compound concepts finally associated to the elements of $\mathcal{P}_{\xi+1}$. The concepts for the classes of $\mathcal{P}_{\xi+1}$ will be: $A_{q}^{\xi+1, X_{k}}=$ $A_{q}^{\xi, X_{k}}, A_{i}^{\xi+1, X_{k}}=\neg A_{q}^{\xi, X_{k}} \wedge B_{i}^{\xi+1, X_{k}}$ and $A_{j}^{\xi+1, X_{k}}=\neg A_{q}^{\xi, X_{k}} \wedge B_{j}^{\xi+1, X_{k}}$

7. Make $\xi=\xi+1$, and return to the step 2) repeating until $\mathcal{P}_{\xi}=\mathcal{P}$.

\section{Results}

The 2-class Partition. For the presented data, $\mathcal{P}_{2}=\left\{C_{393}, C_{392}\right\}$. Figure 2 shows the class-panel graph of $\mathcal{P}_{2}$ [7. As stated in ${ }_{3}$. $I^{k}$ is built using Boxplot 
based discretization (BbD) for all variables. Table 2(left) shows the crossing of $I^{Q-E}$ and $\mathcal{P}_{2}$. Then the $I^{k} \mid \mathcal{P}_{2}$, table is built and totally characterizing variables are identified as those with $I^{k} \mid \mathcal{P}$ table having one 1 per row. Next is to use $B b I R$ to generate all the rules induced for $\mathcal{P}_{2}$. Only rules with $p_{s c}=1$ are considered. Totally characterizing variables are those with $p_{s c}=1$ and $\operatorname{Cov} R=$ $100 \%$ (Q-E, QB-B, QR-G, QP-G, MCRT-B). In this case any of them is useful for conceptualization. Here Q-E is choosen. The Table 3 shown the knowledge Base whit certain rules $\left(p_{s c}=1\right)$ for classer 392 .

Since Q-E is a totally characterizing variable the following concept association can be done:

$-A_{C_{392}}^{2, Q-E}=$ " $x_{Q-E, i} \in[20500.0,23662.9] "$ is associated to $C_{392}$

$-A_{C_{393}}^{2, Q-E}=" x_{Q-E, i} \in[29920.0,54088.6] "$ is associated to $C_{393}$

Or, in other words:

- Class $C_{392}$, "Low values of Inflow wastewater".

- Class $C_{393}$, "High values of Inflow wastewater".

Being low and high defined according to the above numeric limits. Any totally characterizing variable could be used instead. The next step is to go one level down the tree.

The 3-class partition. Take $\mathcal{P}_{3}=\left\{C_{392}, C_{391}, C_{389}\right\}$ and first identify correspondences between the classes of $\mathcal{P}_{2}=\left\{C_{393}, C_{392}\right\}$ and $\mathcal{P}_{3}=\left\{C_{392}, C_{391}, C_{389}\right\}$. Table 2(right) shows the crossing and elicits that class $C_{393}$ splits into $C_{391}$ (referred as $C_{i}^{3}$ in methodology ), $C_{389}$ (referred as $C_{j}^{3}$ in methodology) while $C_{392}$ remains in both $\mathcal{P}_{2}$ and $\mathcal{P}_{3}\left(C_{392}\right.$ is referred as $C_{i}^{2}$ in methodology). From the previous iteration it is already known that there is a common characteristic of both $C_{391}$ and $C_{389}$ (Q-E is greater) which distinguishes them from $C_{392}$ (with lower Q-E). Thus, it is only required to find the separation between $C_{391}$ and $C_{389}$. A similar procedure as the used in previous step for separating $C_{392}$ and $C_{393}$ was used with all the variables regarding $C_{391}$ and $C_{389}$.

Totaly characterizing variables do not exists here. So partially characterizing variables with greater relative covering are considered, in this case SS-D and SSV-D, using variable SS-D for this example. The following system of probabilistic rules than can be induced for the variable SS-D in $\mathcal{P}_{3} \backslash\left\{C_{392}\right\}$ :

$$
\begin{aligned}
& r_{2, \text { classer } 391}^{S S-D}: x_{S S-D, i} \in[63,136] \stackrel{0.8547}{\longrightarrow} \text { classer } 391 \\
& r_{3, \text { classer } 391}^{S-D}: x_{S S-D, i} \in(136,192] \stackrel{1.0}{\longrightarrow} \text { classer } 391, \text { being } B_{391}^{3, S S-D}=x_{S S-D, i} \in(136,192] \\
& r_{1, \text { classer } 389}^{S S-D}: x_{S S-D, i} \in[48.0,63) \stackrel{1.0}{\longrightarrow} \text { classer } 389, \text {, being } B_{389}^{3, S S-D}=x_{S S-D, i} \in[48,63)
\end{aligned}
$$

Combining with the results of previous iteration of $C C E C$ leads on the following interpretation of $\mathcal{P}_{3}$ :

-Class $C_{392}$ is such that "Q-E is in [20500.0, 23662.9]"

-Class $C_{391}$ is such that "Q-E is in [29920.0,54088.6]" and

"SS-D is in $(136,192] " \quad\left(\neg A_{392}^{2, Q-E} \wedge B_{391}^{3, S S-D}\right)$ -Class $C_{389}$ is such that "Q-E is in [29920.0,54088.6]" and

"SS-D is in $[48,63) "$

$$
\left(\neg A_{392}^{2, Q-E} \wedge B_{389}^{3, S S-D}\right)
$$


Table 3. Knowledge Base for classer392

\begin{tabular}{|c|c|c|c|}
\hline Concep. & Rules & Cov & CovR \\
\hline$A_{392}^{2, Q-E}$ & $r_{1, \text { classer } 392}^{Q-E}: x_{Q-E, i} \in[20500.0,23662.9] \stackrel{1.0}{\longrightarrow}$ classer 392 & 6 & $100 \%$ \\
\hline$A_{392}^{2, Q B-B}$ & $r_{1, \text { classer } 392}^{Q B-B}: x_{Q B-B, i} \in[19883.0,22891.0] \stackrel{1.0}{\longrightarrow}$ classer 392 & 6 & $100 \%$ \\
\hline$A_{392}^{2, Q R-G}$ & $r_{1, \text { classer392 }}^{Q R-G}: x_{Q R-G, i} \in[17932.6,18343.5] \stackrel{1.0}{\longrightarrow}$ classer 392 & 6 & $100 \%$ \\
\hline$A_{392}^{2, Q P-G}$ & $r_{1, \text { classer } 392}^{Q P-G}: x_{Q P-G, i} \in[0.0,0.0] \stackrel{1.0}{\longrightarrow}$ classer 392 & 6 & $100 \%$ \\
\hline$A_{392}^{2, Q A-G}$ & $r_{1, \text { classer } 392}^{Q A-G}: x_{Q A-G, i} \in[96451.0,124120.0) \stackrel{1.0}{\longrightarrow}$ classer 392 & 3 & $50 \%$ \\
\hline$A_{392}^{2, S S V-E}$ & $r_{1, \text { classer } 392}^{S S V-E}: x_{S S V-E, i} \in[19.0,30.0) \stackrel{1.0}{\longrightarrow}$ classer 392 & 1 & $16,7 \%$ \\
\hline$A_{392}^{2, D Q O-E}$ & $r_{1, \text { classer } 392}^{D Q O-E}: x_{D Q O-E, i} \in[27.0,100.0) \stackrel{1.0}{\longrightarrow}$ classer 392 & 3 & $50 \%$ \\
\hline$A_{392}^{2, D B O-E}$ & $r_{2, \text { classer } 392}^{D B O-E}: x_{D B O-E, i} \in[73.0,73.0] \stackrel{1.0}{\longrightarrow}$ classer 392 & 1 & $16,7 \%$ \\
\hline$A_{392}^{2, S S-D}$ & $r_{1, \text { classer } 392}^{S S-D}: x_{S S-D, i} \in[40.0,48.0) \stackrel{1.0}{\longrightarrow}$ classer 392 & 2 & $33,33 \%$ \\
\hline$A_{392}^{2, S S V-D}$ & $r_{1, \text { classer } 392}^{S S V-D}: x_{S S V-D, i} \in[13.0,30.0) \stackrel{1.0}{\longrightarrow}$ classer 392 & 3 & $50 \%$ \\
\hline$A_{392}^{2, D Q O-D}$ & $r_{1, \text { classer } 392}^{D Q O-D}: x_{D Q O-D, i} \in[27.0,90.0) \stackrel{1.0}{\longrightarrow}$ classer 392 & 3 & $50 \%$ \\
\hline$A_{392}^{2, D B O-D}$ & $r_{2, \text { classer } 392}^{D B O-D}: x_{D B O-D, i} \in[54.0,54.0] \stackrel{1.0}{\longrightarrow}$ classer 392 & 1 & $16,7 \%$ \\
\hline$A_{392}^{2, M L S S-B}$ & $r_{3, \text { classer } 392}^{\text {MLSS }}: x_{M L S S-B, i} \in(2978.0,3294.0] \stackrel{1.0}{\longrightarrow}$ classer 392 & 2 & $33,33 \%$ \\
\hline$A_{392}^{2, M L V S S-B}$ & $r_{3, \text { classer } 392}^{M L V S S-B}: x_{M L V S S-B, i} \in(2054.0,2100.0] \stackrel{1.0}{\longrightarrow}$ classer 392 & 1 & $16,7 \%$ \\
\hline$A_{392}^{2, M C R T-B}$ & $r_{3, \text { classer } 392}^{M C R T-B}: x_{M C R T-B, i} \in[179.8,341.99] \stackrel{1.0}{\longrightarrow}$ classer 392 & 6 & $100 \%$ \\
\hline
\end{tabular}

Table 4. Knowledge Base for classer389

\begin{tabular}{|c|c|c|c|}
\hline Concep. & Rules & Cov & CovR \\
\hline$B_{389}^{3, Q-E}$ & $r_{3, \text { classer } 389}^{Q-E}: x_{Q-E, i} \in(52255.8,54088.6] \stackrel{1.0}{\longrightarrow}$ classer 389 & 3 & $4,28 \%$ \\
\hline$B_{389}^{3, Q B-B}$ & $r_{3, \text { classer } 389}^{Q B-B}: x_{Q B-B, i} \in(49695.8,52244.6] \stackrel{1.0}{\longrightarrow}$ classer 389 & 3 & $4,28 \%$ \\
\hline$B_{389}^{3, Q R-G}$ & $r_{1, \text { classer } 389}^{Q R-G}: x_{Q R-G, i} \in[26218.0,27351.0) \stackrel{1.0}{\longrightarrow}$ classer 389 & 2 & $2,85 \%$ \\
\hline$B_{389}^{3, Q P-G}$ & $r_{1, \text { classer } 389}^{Q P-G}: x_{Q P-G, i} \in[188.0,327.6) \stackrel{1.0}{\longrightarrow}$ classer 389 & 2 & $2,85 \%$ \\
\hline$B_{389}^{3, Q A-G}$ & $r_{1, \text { classer } 389}^{Q A-G}: x_{Q A-G, i} \in[124120.0,136371.0) \stackrel{1.0}{\longrightarrow}$ classer 389 & 2 & $2,85 \%$ \\
\hline$B_{389}^{3, P H-E}$ & $r_{3, \text { classer } 389}^{P H-E}: x_{P H-E, i} \in(7.9,8.0] \stackrel{1.0}{\longrightarrow}$ classer 389 & 1 & $1,42 \%$ \\
\hline$B_{389}^{3, S S-E}$ & $r_{1, \text { classer } 389}^{S S-E}: x_{S S-E, i} \in[62.0,82.0) \stackrel{1.0}{\longrightarrow}$ classer 389 & 1 & $1,42 \%$ \\
\hline \begin{tabular}{|l}
,$S S V-E$ \\
389
\end{tabular} & $r_{1, \text { classer } 389}^{S S V-E}: x_{S S V-E, i} \in[30.0,60.0) \stackrel{1.0}{\longrightarrow}$ classer 389 & 2 & $2,85 \%$ \\
\hline$B_{389}^{3, D Q O-E}$ & $r_{1, \text { classer } 389}^{D Q O-E}: x_{D Q O-E, i} \in[100.0,158.0) \stackrel{1.0}{\longrightarrow}$ classer 389 & 1 & $1,42 \%$ \\
\hline$B_{389}^{3, D B O-E}$ & $r_{1, \text { classer } 389}^{D B O-E}: x_{D B O-E, i} \in[69.0,90.0) \stackrel{1.0}{\longrightarrow}$ classer 389 & 5 & $7,14 \%$ \\
\hline$B_{389}^{3, S S-D}$ & $r_{1, \text { classer } 389}^{S S-D}: x_{S S-D, i} \in[48.0,63.0) \stackrel{1.0}{\longrightarrow}$ classer 389 & 24 & $34,28 \%$ \\
\hline$B_{389}^{3, S S V-D}$ & $r_{1, \text { classer } 389}^{S S V}: x_{S S V-D, i} \in[30.0,47.0) \stackrel{1.0}{\longrightarrow}$ classer 389 & 25 & $35,71 \%$ \\
\hline$B_{389}^{3, D B O-D}$ & $r_{1, \text { classer } 389}^{D B O-D}: x_{D B O-D, i} \in[36.0,56.0) \stackrel{1.0}{\longrightarrow}$ classer 389 & 5 & $7,14 \%$ \\
\hline$B_{389}^{3, D B O-S}$ & $r_{1, \text { classer } 389}^{D B O-S}: x_{D B O-S, i} \in[2.0,4.0) \stackrel{1.0}{\longrightarrow}$ classer 389 & 2 & $2,85 \%$ \\
\hline$B_{389}^{3, M L S S-B}$ & $r_{3, \text { classer } 389}^{\text {MLSS }}: x_{M L S S-B, i} \in(2696.0,2978.0] \stackrel{1.0}{\longrightarrow}$ classer 389 & 1 & $1,42 \%$ \\
\hline$B_{389}^{3, M C R T-B}$ & $r_{3, \text { classer } 389}^{M C R T-B}: x_{M C R T-B, i} \in(28.8,34.4] \stackrel{1.0}{\longrightarrow}$ classer 389 & 1 & $1,42 \%$ \\
\hline
\end{tabular}

Since anyone of these interpretations is based on non totally characterizing variables, some degree of certainty should be associated depending on the probabilities of the corresponding generated concepts. The interpretation with the highest global degree of certainty is the one that intends to be refined in the following iteration. Table 4 showns the Knowledge Base with certain rules $\left(p_{s c}=1\right)$ induced for classer 389 . 
Table 5. Knowledge Base for classer383

\begin{tabular}{|l|l|c|c|}
\hline Concep. & Rules & Cov & Cov $R$ \\
\hline$\neg C_{390}^{4, S S-E}$ & $r_{3, \text { classer } 383}^{S S-E}: x_{S S-E, i} \in(480.0,655.0] \stackrel{1.0}{\longrightarrow}$ classer 383 & 5 & $14,70 \%$ \\
\hline$\neg C_{390}^{4, S S V-E}$ & $r_{3, \text { classer } 383}^{S S}: x_{S S V-E, i} \in(336.0,593.0] \stackrel{1.0}{\longrightarrow}$ classer 383 & 4 & $11,76 \%$ \\
\hline$\neg C_{390}^{4, D Q O-E}$ & $r_{3, \text { classer } 383}^{D Q O-E}: x_{D Q O-E, i} \in(1279.0,1579.0] \stackrel{1.0}{\longrightarrow}$ classer 383 & 1 & $2,94 \%$ \\
\hline$\neg C_{390}^{4, D B O-E}$ & $r_{3, \text { classer } 383}^{D B-E}: x_{D B O-E, i} \in(382.0,987.0] \stackrel{1.0}{\longrightarrow}$ classer 383 & 7 & $20,58 \%$ \\
\hline$\neg C_{390}^{4, M L V S S-B}$ & $r_{1, \text { classer } 383}^{M L S S}: x_{M L V S S-B, i} \in[185.0,611.0) \stackrel{1.0}{\longrightarrow}$ classer 383 & 2 & $5,88 \%$ \\
\hline$\neg C_{390}^{4, M C R T-B}$ & $r_{1, \text { classer } 383}^{M C R T}: x_{M C R T-B, i} \in[1.8,6.2) \stackrel{1.0}{\longrightarrow}$ classer 383 & 1 & $2,94 \%$ \\
\hline
\end{tabular}

The final partition. The process would continue separating the classes $C_{390}$ and $C_{383}$ of the following partition $\mathcal{P}_{4}$, see figure 1(left), which are the subdivision of $C_{391}$. Similarly, the following interpretation of $\mathcal{P}_{4}$, which is the final partition [4] is obtained with the following Knowledge Base with certain rules for classer383(Table 5) and classer390 (Table 6):

And de final interpretation is:

- Class $C_{392}$ is such that $A_{392}^{2, Q-E}$

- Class $C_{389}$ is such that $\neg A_{392}^{2, Q-E} \wedge B_{391}^{3, S S-D}$

- Class $C_{390}$ is such that $\neg A_{392}^{2, Q-E} \wedge \neg B_{391}^{3, S S-D} \wedge C_{390}^{4, D B O-E}$

- Class $C_{383}$ is such that $\neg A_{392}^{2, Q-E} \wedge \neg B_{391}^{3, S S-D} \wedge \neg C_{390}^{4, D B O-E}$. Or, in other words:

- Class $C_{392}$, "Low values for Inflow wastewater"

- Class $C_{389}$, "High values for Inflow and few Suspended solids at the settler"

- Class $C_{390}$, "High values of Inflow wastewater, high Suspended solids at the exit and few Biodegradable organic matter at the input"

- Class $C_{383}$, "Medium-high values of Inflow wastewater, high Suspended solids at the settler and high Biodegradable organic matter at the input."

This set of rules can, in fact, be considered as a domain model which can support later decision on the treatment to be applied to a new day, provided that a standard treatment is previously associated to every class by experts. In this association the possibility of easily interpreting the classes is critical as well as to provide the experts means for easily understanding the meaning of the classes. In this sense the proposed method provides simple and short rules which use to be easier to handle than those provided by other induction rules algorithms. This final interpretation is consistent with the one provided by the experts and presented in [4].

\section{Conclusions and Future Work}

In this paper the first approach of a methodology to generate automatic interpretations from a group of classes is presented. Concepts associated to classes are built taking advantage of hierarchical structure of the underlying clustering. The Conceptual characterization by embedded conditioning CCEC [2] is a quick and effective method that generates a conceptual model of the domain, which will be from great support to the later decision making based on a combination 
Table 6. Knowledge Base for classer390

\begin{tabular}{|c|c|c|c|}
\hline Concep. & Rules & Cov & $\operatorname{Cov} R$ \\
\hline$C_{390}^{4, Q-E}$ & $r_{1, \text { classer } 390}^{Q-E}: x_{Q-E, i} \in[29920.0,34284.4) \stackrel{1.0}{\longrightarrow}$ classer 390 & 9 & $3,15 \%$ \\
\hline $\begin{array}{ll}4, Q B-B \\
390\end{array}$ & $r_{3, \text { classer } 390}^{Q B-B}: x_{Q B-B, i} \in(39000.0,49695.8] \stackrel{1.0}{\longrightarrow}$ classer 390 & 60 & $21,06 \%$ \\
\hline$C_{390}^{4, Q R-G}$ & $r_{3, \text { classer } 390}^{Q R-G}: x_{Q R-G, i} \in(44568.6,49527.0] \stackrel{1.0}{\longrightarrow}$ classer 390 & 13 & $4,56 \%$ \\
\hline$C_{390}^{4, Q P-G}$ & $r_{3, \text { classer } 390}^{Q P-G}: x_{Q P-G, i} \in(831.1,1080.0] \stackrel{1.0}{\longrightarrow}$ classer 390 & 56 & $19,64 \%$ \\
\hline$C_{390}^{4, Q A-G}$ & $r_{1, \text { classer } 390}^{Q A-G}: x_{Q A-G, i} \in[136371.0,156320.0) \stackrel{1.0}{\longrightarrow}$ classer 390 & 7 & $2,45 \%$ \\
\hline$C_{390}^{4, F E-E}$ & $r_{3, \text { classer } 390}^{F E-E}: x_{F E-E, i} \in(63.3,89.8] \stackrel{1.0}{\longrightarrow}$ classer 390 & 7 & $2,45 \%$ \\
\hline$C_{390}^{4, P H-E}$ & $r_{3, \text { classer } 390}^{P H-E}: x_{P H-E, i} \in(7.8,7.9] \stackrel{1.0}{\longrightarrow}$ classer 390 & 8 & $2,80 \%$ \\
\hline$C_{390}^{4, S S-E}$ & $r_{1, \text { classer } 390}^{S S-E}: x_{S S-E, i} \in[82.0,114.0) \stackrel{1.0}{\longrightarrow}$ classer 390 & 4 & $1,40 \%$ \\
\hline$C_{390}^{4, S S V-E}$ & $r_{1, \text { classer } 390}^{S S V-E}: x_{S S V-E, i} \in[60.0,92.0) \stackrel{1.0}{\longrightarrow}$ classer 390 & 7 & $2,45 \%$ \\
\hline$C_{390}^{4, D Q O-E}$ & $r_{1, \text { classer } 390}^{D Q O-E}: x_{D Q O-E, i} \in[158.0,414.0) \stackrel{1.0}{\longrightarrow}$ classer 390 & 113 & $39,64 \%$ \\
\hline$C_{390}^{4, D B O-E}$ & $r_{1, \text { classer } 390}^{D B O-E}: x_{D B O-E, i} \in[90.0,220.0) \stackrel{1.0}{\longrightarrow}$ classer 390 & 126 & $44,21 \%$ \\
\hline$C_{390}^{4, P H-D}$ & $r_{1, \text { classer } 390}^{P H-D}: x_{P H-D, i} \in[7.2,7.3) \stackrel{1.0}{\longrightarrow}$ classer 390 & 3 & $1,05 \%$ \\
\hline$C_{390}^{4, S S-D}$ & $r_{3, \text { classer } 390}^{\text {SS-D }}: x_{S S-D, i} \in(112.0,192.0] \stackrel{1.0}{\longrightarrow}$ classer 390 & 30 & $10,52 \%$ \\
\hline$C_{390}^{4, S S V-D}$ & $r_{3, \text { classer } 390}^{S S V-D}: x_{S S V-D, i} \in(92.0,134.0] \stackrel{1.0}{\longrightarrow}$ classer 390 & 11 & $3,85 \%$ \\
\hline$C_{390}^{4, D Q O-D}$ & $r_{3, \text { classer } 390}^{D Q O-D}: x_{D Q O-D, i} \in(329.0,538.0] \stackrel{1.0}{\longrightarrow}$ classer 390 & 22 & $7,71 \%$ \\
\hline$C_{390}^{4, D B O-D}$ & $r_{3, \text { classer } 390}^{D B O-D}: x_{D B O-D, i} \in(224.0,274.0] \stackrel{1.0}{\longrightarrow}$ classer 390 & 4 & $1,40 \%$ \\
\hline$C_{390}^{4, P H-S}$ & $r_{3, \text { classer } 390}^{P H-S}: x_{P H-S, i} \in(7.8,8.0] \stackrel{1.0}{\longrightarrow}$ classer 390 & 16 & $5,61 \%$ \\
\hline$C_{390}^{4, S S-S}$ & $r_{3, \text { classer } 390}^{S S-S}: x_{S S-S, i} \in(29.0,174.8] \stackrel{1.0}{\longrightarrow}$ classer 390 & 25 & $8,77 \%$ \\
\hline$C_{390}^{4, S S V-S}$ & $r_{3, \text { classer } 390}^{S S V-S}: x_{S S V-S, i} \in(19.0,134.8] \stackrel{1.0}{\longrightarrow}$ classer 390 & 38 & $13,3 \%$ \\
\hline \begin{tabular}{|l|l}
$C_{390}^{4, D Q O-S}$ \\
\end{tabular} & $S, i \in(95.0,163.0] \stackrel{1.0}{\longrightarrow}$ classer 390 & 24 & $8,4 \%$ \\
\hline$C_{390}^{4, D B O-S}$ & $r_{3, \text { classer } 390}^{D B O-S}: x_{D B O-S, i} \in(35.0,84.0] \stackrel{1.0}{\longrightarrow}$ classer 390 & 4 & $1,40 \%$ \\
\hline$C_{390}^{4, V 30-B}$ & $r_{1, \text { classer } 390}^{V 30-B}: x_{V 30-B, i} \in[77.0,140.0) \stackrel{1.0}{\longrightarrow}$ classer 390 & 26 & $9,12 \%$ \\
\hline$C_{390}^{4, M L S S-B}$ & $r_{3, \text { classer } 390}^{M L S S-B}: x_{M L S S-B, i} \in(2248.0,2696.0] \stackrel{1.0}{\longrightarrow}$ classer 390 & 17 & $5,96 \%$ \\
\hline$C_{390}^{4, M L V S S-B}$ & $r_{3, \text { classer } 390}^{M L V S}: x_{M L V S S-B, i} \in(1726.0,2054.0] \stackrel{1.0}{\longrightarrow}$ classer 390 & 18 & $6,31 \%$ \\
\hline $\begin{array}{l}, M C R T-B \\
390\end{array}$ & $r_{3, \text { classer } 390}^{M C R T-B}: x_{M C R T-B, i} \in(16.0,28.8] \stackrel{1.0}{\longrightarrow}$ classer 390 & 8 & $2,81 \%$ \\
\hline
\end{tabular}

of boxplop based discretization and an interactive combination of concepts upon hierarchical subdivisions of the domain. This is a preliminary proposal that has been applied with success to real data coming from a WWTP. Benefits of those proposal are specially interesting in the interpretation of partitions with great number of classes. Automatic generation of interpretations cover the important goal of KD of describing the domain [11. However, in this proposal a direct connection between the generated concepts and the automatic rules generation allows direct construction of a decision model for the domain oriented to later class prediction. As a matter of fact, automatic production of probabilistic or fuzzy classification rules regarding concepts provided by $C C E C$ is direct, as discussed in [12. By associating an appropriate standard treatment to every class a model for deciding the appropriate treatment of a concrete day upon a reduced number variables is obtained together with an estimation of the risk associated to that decision (which is related with the certainty of the rule). At present other automatic criteria for deciding which variable is kept at every iteration (here the covering of the all concepts was used) and how to propagate uncertainty from 
one iteration to the next is in progress (here rules with $p_{s c}=1$ are used). The idea is to use an approach which avoids the explicit construction of all the concepts to evaluate their coverage. Comparison of rules produced with $C C E C$ and other inductive methods ID3-like is also in progress, as well as validation with a second real WWTP from Slovenia.

\section{References}

1. Gordon, A.D.: Identifying genuine clusters in a classification. Computational Statistics and Data Analysis 18, 561-581 (1994)

2. Gibert, K., Pérez-Bonilla, A.: Ventajas de la estructura jerárquica del clustering en la interpretración automática de clasificaciones. In: III TAMIDA, pp. 67-76 (2005)

3. Gibert, K.: The use of symbolic information in automation of statistical treatment for ill-structured domains. AI Communications 9, 36-37 (1996)

4. Gibert, K., Roda, I.: Identifying characteristic situations in wastewater treatment plants. In: Workshop BESAI (ECAI), vol. 1, pp. 1-9 (2000)

5. Gibert, K., Aluja, T., Cortés, U.: Knowledge Discovery with Clustering Based on Rules. Interpreting results. In: Żytkow, J.M. (ed.) PKDD 1998. LNCS, vol. 1510, pp. 83-92. Springer, Heidelberg (1998)

6. Metcalf, E.: Wastewater engineering treatament. Disposal and reuse. McGraw-Hill 4th edn. revised by George Tchobanoglous, Franklin L. Burton NY.US (2003)

7. Gibert, K., Nonell, et al.: Knowledge discovery with clustering: impact of metrics and reporting phase by using klass. Neural Network World 4/05, 319-326 (2005)

8. Tukey, J.: Exploratory Data Analysis. Addison-Wesley, Reading (1977)

9. Gibert, K.: Técnicas híbridas de Inteligencia Artificial y Estadística para el descubrimiento de conocimiento y la minería de datos. In: Thompson (ed.) Tendencias de la minería de datos en España, pp. 119-130 (2004)

10. Gibert, K., Pérez-Bonilla, A.: Revised boxplot based discretization as a tool for automatic interpretation of classes from hierarchical cluster. In: Series Studies in Classification, D.A., pp. 229-237. Springer, Heidelberg (2006)

11. Fayyad, U., et al.: From Data Mining to Knowledge Discovery: An overview. In: Advances in Knowledge Discovery and Data Mining, AAAI/MIT Press (1996)

12. Gibert, K., Pérez-Bonilla, A.: Taking advantage of the hierarchical structure of a clustering for automatic generation of classification interpretations. In: 4th EUSFLAT, España, pp. 524-529 (2005) 